gimes. The influence of the open method of coordination on national reforms. New York: Routledge, 37-60.

Weishaupt, Timo (2009): Money, Votes or „Good“ Ideas? Partisan Politics and the Effectiveness of the European Employment Strategy in Austria and Ireland, in: Kröger, Sandra (ed.), What we have learnt: Advances, pitfalls and remaining questions of OMC research. European Integration Online Papers, special issue 1 (13).

Zeitlin, Jonathan (2005): Conclusion, in: Zeitlin, Jonathan/ Pochet, Philippe/Magnusson, Lars (eds), The Open Method of Coordination in Action: The European Employment and Social Inclusion Strategies. Brussels: Peter Lang, 441-498.

- (2009): The Open Method of Coordination and reform of national social and employment policies: influences, mechanisms, effects, in Heidenreich, Martin / Zeitlin, Jonathan (eds.), Changing European Employment and Welfare Regimes: The Influence of the Open Method of Coordination on National Reforms. Oxon: Routledge, 214-245.

\section{Warum betreibt der EuGH Rechtsfortbildung?}

\section{Die Politisierungshypothese ${ }^{1}$}

\section{Martin Höpner}

\section{Zusammenfassung}

In der rechtswissenschaftlichen, politologischen und soziologischen Fachliteratur ist unumstritten, dass der EuGH das europäische Recht als „Motor der Integration“ expansiv interpretiert und damit faktisch Integrationspolitik betreibt. Der Beitrag diskutiert anhand von zehn Richtungsentscheidungen des EuGH, ob die Politisierungshypothese einen Beitrag zur Erklärung dieses Umstands leisten kann. Die Hypothese besagt, dass EuGHRichter ihre Entscheidungen auf Grundlage von länderspezifischen oder parteipolitischen Interessen fällen. Der parteienbezogenen Variante der Hypothese scheint keine Erklärungskraft zuzukommen. Auch die länderbezogene Variante vermag keine systematische Erklärung der EuGH-Rechtsfortbildung zu leisten, die an die Stelle konkurrierender Deutungen treten könnte. Auf ausgewählte Fälle aber passt die länderbezogene Hypothese recht gut. Auf Grundlage der vorgefundenen Ergebnisse formuliere ich die These, dass situative Akte der Politisierung des EuGH möglich erscheinen, ohne dass der rechtliche Code bei der Entscheidungsfindung damit vollständig und dauerhaft vom politischen Code verdrängt würde.

\section{Abstract: Why Does the ECJ Augment European Law? The Politicization Hypothesis}

Scholars from jurisprudence, political science and sociology agree that the ECJ interprets European law extensively and that the Court has become an ,engine of integration' that, de facto, conducts integration policy. On the basis of ten landmark decisions of the ECJ, this article discusses whether the politicization hypothesis helps to explain this outcome. The politicization hypothesis predicts that ECJ judges make decisions in line with political interests of either the countries they come from or the parties they sympathize with. There seems to be no explanatory power of the party-based variant of the hypothesis. Similarly, the country-based variant of the hypothesis delivers no systematic explanation that could supersede alternative explanations. How- ever, the country-based variant fits pretty well with some of the observed cases. The article concludes that situational politicization constellations may actually occur at the ECJ, without entirely or permanently replacing the judicial with the political code.

\section{Richterliche Fortbildung des Europarechts als Gegenstand politikwissenschaftlicher Forschung}

Bereits seit den sechziger Jahren weist die Rechtsprechung des Europäischen Gerichtshofs (EuGH) einen Bias im Sinne einer dezidiert proeuropäischen Judikatur auf. Dieser Bias der Rechtsprechung beschränkt sich nicht auf die maximal integrationsfreundliche Auslegung bestehender Grundsätze im europäischen Primär- und Sekundärrecht und die damit einhergehende Ausweitung des Wirkungskreises des europäischen Rechts zuungunsten der mitgliedsstaatlichen Rechtsordnungen. Vielmehr sind zahlreiche europarechtliche Grundsätze selbst Schöpfungen des EuGH. Dazu zählen der Vorrang und die Direktwirkung des europäischen Rechts, dessen unmittelbare Drittwirkung auf Private (beispielsweise Unternehmen und Verbände), die spezifische normative Signifikanz der Grundfreiheiten als Beschränkungsverbote (statt lediglich: Diskriminierungsverbote) und europäische Grundrechte als allgemeine Rechtsgrundsätze der Gemeinschaft. Mit anderen Worten: Der EuGH hat sich vom „Hüter der Verträge“ zum „Motor der Integration“ entwickelt und betreibt Rechtsfortbildung und -schöpfung, ${ }^{2}$ die sich im Ergebnis nicht von politischen Vertragsrevisionen unterscheidet. Dieser Befund ist in der politikwissenschaftlichen, der rechtswissenschaftlichen und der soziologischen Forschungsliteratur unumstritten (siehe etwa Alter 2001; Höreth 2008: Abschnitt II 1.; Münch 2008; Weiler 2004; sowie die Beiträge in Roth/Hilpold 2008).

Drei Folgewirkungen des „Pro-Europa-Dralls“ der Rechtsprechung lassen sich unterscheiden (Scharpf 2009). Erstens: Die europäische Integration wird stärker beschleunigt, als es von den Mitgliedstaaten zu den Zeitpunkten der Vertragsschlüsse und -revisionen sowie der Verabschiedung des von Rechtsfortbildung betroffenen Sekundärrechts beabsichtigt war. Die integrationsfreundliche Rechtsprechung bewirkt, dass die Entscheidung der Mitgliedstaaten, eine Kompetenz an die europäischen Ebene zu delegieren, intensiver geschützt wird als die Entscheidung, eine Kompetenz weiterhin autonom ausüben zu wollen obwohl doch beide gleichermaßen des Schutzes durch das europäische Höchstgericht bedürfen. Daraus folgt ein Problem mangelnder demokratischer Steuerbarkeit des Integrationsprozess, also: ein Demokratiedefizit. Zweitens: Die systematische Neigung zur rechtlich betriebenen Integrationspolitik verschiebt die Balance zwischen Recht und Politik. Zwar sind Spannungsverhältnisse zwischen Recht und Politik eine gewissermaßen „,natürliche“ Begleiterscheinungen aller politischen Ordnungen, in denen Legislative und Exekutive ein unabhängiges Verfassungsgericht an die Seite gestellt wird (grundlegend Dahl 1957; jüngst beispielsweise Kneip 2009). Dieses Problem stellt sich auf europäischer Ebene jedoch mit größerer Brisanz als in den Nationalstaaten, weil die politische Korrigierbarkeit der EuGHRechtsprechung gegen Null tendiert (ausführlich: Höpner 2010). Drittens: Die extensive EuGH-Rechtsprechung ist nicht „politisch neutral“", ob sie will oder nicht. Denn sie kann nationale marktkorrigierende Institutionen dem Wirkungskreis von

1 Ich danke Michael Blauberger, Philip Mader, Florian Rödl, Armin Schäfer, Susanne K. Schmidt, Wolfgang Streeck und den anonymen Gutachtern der Zeitschrift „Sozialer Fortschritt“ für hilfreiche Hinweise.

2 In diesem Text verwende ich den Terminus der Rechtsfortbildung in Abgrenzung vom Terminus der Lückenfüllung. Richterliche Rechtsfortbildung liegt vor, wen Richterrecht eine Anreicherung des Primär- oder Sekundärrechts hervorbringt, die über die Füllung von sowohl systemwidrigen als auch gegen die Intention des Gesetzgebers bestehenden Rechtslücken hinausgeht (vergleiche hierzu ausführlich Höpner 2010). 
Grundfreiheiten und europäischem Wettbewerbsrecht unterstellen und damit liberalisierend wirken. Im Zuge einer kraftvollen Rechtsprechung zur Antidiskriminierung kann sie die Marktzugangsrechte Benachteiligter stützen und beispielsweise auf Nichtdiskriminierung am Arbeitsplatz hinwirken. Die Schaffung marktkorrigierender Institutionen mit umverteilender Funktion ist auf europäischer Ebene durch eine noch so expansive Rechtsprechung aber nicht möglich. Marktkorrigierende Institutionen verharren somit auf Ebene der Mitgliedstaaten und laufen zunehmend Gefahr, vom EuGH als Beschränkungen der Marktfreiheiten verworfen zu werden. Aus diesem Grund geht mit der Rechtsfortbildung ein systematischer liberalisierungspolitischer Bias einher.

Neben den Wirkungen haben auch die Ursachen europäischer Rechtsfortbildung das Interesse der Integrationsforschung geweckt. In dieser Hinsicht lassen sich strukturelle und akteursbezogene Erklärungen unterscheiden. Strukturelle Erklärungen verweisen auf das institutionelle, organisatorische und politökonomische Umfeld, in dem sich die Rechtsprechung des EuGH vollzieht (Höreth 2008: Kapitel V; Scharpf 2009). Im Kern besagen sie, dass bemerkenswert niedrige Hürden der Rechtsfortbildung durch EuGH-Richter bemerkenswert hohen Hürden der Mitgliedstaaten, sich koordiniert gegen die expansive Judikatur zu wehren, gegenüberstehen.

So können einmal berufene Richter während ihrer sechs Jahre dauernden Amtszeit von dem Mitgliedstaaten nicht wieder abgesetzt werden (zur Einführung in die institutionellen Grundlagen des EuGH: Wessels 2008: Kapitel III.5; Pollack 1997). Die Beratungen der Kammern erfolgen geheim. Anders als etwa beim deutschen Bundesverfassungsgericht oder dem U.S. Supreme Court werden den Urteilen keine namentlich gekennzeichneten Minderheitsvoten angefügt (,dissenting opinions“), so dass das Verhalten einzelner Richter durch die Mitgliedstaaten faktisch nicht kontrolliert werden kann. Die Entscheidungen des Gerichtshofs beruhen auf der einfachen Mehrheitsregel, so dass Blockaden aufgrund eines Einstimmigkeitserfordernisses auszuschließen sind. EuGH-Entscheidungen unterliegen keiner weitergehenden judiziellen Kontrolle, etwa durch einen mitunter geforderten Kompetenzgerichtshof. Der EuGH fungiert bei Kompetenzstreitigkeiten zwischen europäischen Organen und Mitgliedstaaten als Höchstgericht und tritt damit, weil er selbst zu den europäischen Organen zählt, ,letztentscheidend als Richter in eigener Sache“ (Dobler 2008: 529) auf. Dem gegenüber setzt die Änderung dieser institutionellen Grundlagen politisch herbeizuführende Vertragsänderungen voraus. Die Drohung mit Sanktionen gegen weitgehende Schritte richterlicher Rechtsfortbildung ist damit faktisch unglaubwürdig, ${ }^{3}$ kann vom Gerichtshof ignoriert werden und lässt dem Ermessensspielraum europäischer Richter, Integrationspolitik zu betreiben, weite Spielräume.

Nun erklären die strukturell determinierten weiten Handlungsspielräume noch nicht, warum sie durch die Rechtsprechung auch tatsächlich genutzt werden. Vor allem erklären sie nicht, warum sie nahezu ausschließlich in ein- und derselben, nämlich: integrationsvertiefenden Richtung ausgefüllt werden, wäre doch im Prinzip auch denkbar, dass die von den Mitgliedstaaten an den EuGH entsandten Richter die weiten Handlungsspielräume nutzen, um europäische Kompetenzen an die Mitgliedstaaten rückzuverlagern. Hier kommen akteursbezogen-soziologische Erklärungen ins Spiel. An anderer Stelle ${ }^{4}$ schlage ich ein zweistufiges Modell vor, das - kürzest möglich formuliert - mit folgenden Annahmen operiert. Erstens: Die an den EuGH berufenen Richter sind von spezifischen Eigenarten des europarechtlichen Diskurses geprägt. Das Europarecht (als Profession) hat sich aus dem Völkerrecht entwickelt. Das Völkerrecht bewegt sich in Opposition zum Souveränitätsgedanken der klassischen Staatsrechtslehre und trägt den normativen Anspruch in sich, zur Entstehung verbindlicher Ordnungen auf supranationaler Ebene beitragen und diese rechtfertigen zu wollen. Im Vergleich zum klassischen Staatsrecht stellt sich im Völkerrecht die Frage der demokratischen Legitimation normativer
Ordnungen weniger, denn es verfolgt Ziele wie die Einhegung konflikthafter internationaler Beziehungen, über die im Prinzip Einigkeit herrscht. Zudem existiert im Völkerrecht wenig geschriebenes Recht. Die Entstehung normativer Ordnungen auf supranationaler Ebene erfordert den kreativen, rechtsschöpfenden Juristen. Und zweitens: Innerhalb des EuGH erfolgt die Zuschreibung von Status über die Beteiligung an spektakulären und mutigen, gleichwohl natürlich: juridisch möglichst unangreifbaren Urteilen. Das Streben nach Mitwirkung an der schrittweisen Verwirklichung visionärer Integrationsziele fällt im Luxemburger Gericht auf fruchtbaren Boden.

Es besteht kein Anlass, strukturelle und akteursbezogen-soziologische Erklärungen gegeneinander auszuspielen. Sie sind vereinbare, komplementäre Erklärungen und beruhen auf einund derselben Problemdeutung. Auf diesen Diskussionsstand aufbauend, diskutiere ich nachfolgend ein abweichendes Erklärungsangebot, das ich mit dem Begriff der „Politisierungshypothese" umschreibe.

\section{Die Politisierungshypothese}

Die Politisierungshypothese hält eine alternative Erklärung der ,aktivistischen“ EuGH-Rechtsprechung bereit. Sie besagt, dass der EuGH bei politisch brisanten Richtungsentscheidungen zur Aushandlungsarena divergierender politischer Interessen mutiert. Solche Interessen können entlang von Länderoder Parteiengrenzen verlaufen. Der länderbezogenen Variante der Hypothese zufolge entsteht Integrationspolitik durch Rechtsfortbildung, wenn sich in der mit der Rechtsfindung betrauten Kammer des Gerichts eine Mehrheit an Richtern aus Ländern findet, die ein Interesse an der durch die Urteile bewirkten Integrationspolitik haben (beispielsweise, weil sie von der bewirkten wirtschaftlichen Liberalisierung oder etwa dem transnationalen Zugang zu den sozialen Sicherungssystemen der Mitgliedstaaten in asymmetrischer Weise profitieren). Analog hierzu besagt die parteienbezogene Variante der Hypothese, dass Integrationspolitik durch Rechtsfortbildung entsteht, wenn die Urteile von Richtern gefällt werden, die Parteien nahestehen, deren Präferenzen die entsprechenden Entscheidungen entgegenkommen (beispielsweise, weil die parteipolitischen $\mathrm{Zu}-$ gehörigkeiten der Richter eine Präferenz für wirtschaftliche Liberalisierung oder für die Stärkung von Arbeitnehmerrechten nahelegen). ${ }^{5}$

Zur Illustration wähle ich ein Zitat des Gewerkschaftsfunktionärs und Publizisten Michael Wendl, das den Kern der Hypothese besonders klar zum Ausdruck bringt. ${ }^{6}$ Referenzpunkt des Autors sind die Urteile in den Fällen Laval und Rüffert, auf die ich weiter unten noch einmal zurückkommen werde. Diese Urteile erregten Aufsehen, weil sie der Fähigkeit einzelner Mitgliedstaaten, eigene Arbeitsstandards auf zeitweilig aus anderen EU-

3 Jeder einzelne der 27 Mitgliedstaaten, der von der Stoßrichtung der EuGH-Rechtsprechung in asymmetrischer Weise profitiert, kann Reformen der institutionellen Grundlagen des Gerichts im Prinzip verhindern.

4 In Vorbereitung.

5 Ich danke für Michael Blauberger für den zutreffenden Hinweis, dass es stets der Kombination mit weiteren Hypothesen bedarf, um zu erklären, warum der hohen Anzahl integrationsfreundlicher EuGH-Urteile keine ebenso hohe Anzahl an Urteilen gegenüber steht, die Kompetenzen von den europäischen Organen an die Mitgliedstaaten rückverlagern. Auch falls sich also die vom EuGH betriebene Integrationspolitik in systematischer Weise auf günstige politische Konstellationen innerhalb der Richtergremien zurückführen ließe, bliebe zu klären, warum Akte der Politisierung nicht auch in umgekehrter, der Integration Grenzen setzender Richtung wirken. Meines Erachtens ist die Existenz der integrationspolitischen „Einbahnstraße" vor allem akteursbezogen-soziologisch, unter Rückgriff auf die proeuropäischen Visionen supranationaler europäischer Akteure, erklärbar (also unter Verweis auf Variablen außerhalb der Politisierungshypothese im Sinne dieser Studie).

6 Eine vergleichbare Fundstelle, die ebenfalls auf das Laval-Urteil verweist, ist Robert (2009). 
Staaten entsandte Arbeitnehmer zu übertragen, überraschend enge Grenzen setzten (im Einzelnen: Höpner 2008).

Wendl (2008) interpretiert die Urteile wie folgt:

„Die ... Urteile waren knappe Mehrheitsentscheidungen, die zustande gekommen sind, weil Richter aus den neuen Beitrittsländern der EU, also aus Osteuropa, den Ausschlag gegeben haben. ... Aus meiner Sicht haben die osteuropäischen Richter politisch zugunsten osteuropäischer Arbeitsmigranten und deren zentralem Wettbewerbsvorteil, den niedrigen Löhnen, entschieden. Wenn für polnische oder lettische Arbeiter deutsche oder schwedische Löhne gezahlt werden müssen, verlieren diese Arbeiter ihren entscheidenden Vorteil im Verdrängungsprozess auf dem europäischen Arbeitsmarkt. Wenn das der materielle Kern des juristischen Streits ist, dann haben innerjuristische Auseinandersetzungen zunächst wenige Aussichten auf Erfolg. In der Frage der politischen und rechtlichen Auslegung des europäischen Rechts ist diese Ebene wichtig, aber wenn gegensätzliche materielle Interessen in Konflikt geraten, ist die Überzeugungskraft des besseren juristischen Arguments gering."

In der Literatur über nationale Verfassungsgerichte hat die Politisierungshypothese eine lange Tradition (grundlegend: Pritchett 1948), wobei die Politisierung hier aber vor allem entlang der parteipolitischen Links-Rechts-Achse, nicht entlang der regionalen Herkunft der Richter gedacht wird. Neben theoretischen Herleitungen (van Hees/Steunenberg 2000) finden sich auch empirische Tests der Hypothese. So hat Hönnige (2007: Kapitel 6) jüngst anhand des deutschen Beispiels überzeugend nachgewiesen, dass die parteipolitische Nähe von Verfassungsrichtern für deren Handeln einen systematischen Unterschied macht: „Richter lassen ihre politischen Einstellungen in ihre Urteile einfließen und das Urteil hängt von der politischen Besetzung der Richterbank ab" (ebd.: 199). Ähnliche Befunde liegen beispielsweise für Portugal und die Niederlande vor (vergleiche die bei Hönnige 2007: 71 zitierte Literatur). Für den EuGH ist die Politisierungshypothese meines Wissens bisher nicht empirisch überprüft worden.

Auch die in Abschnitt 1 vorgestellten Erklärungen richterlicher Rechtsfortbildung operierten mit der Annahme, der EuGH betreibe faktisch, aus welchen Gründen auch immer, Integrationspolitik. In diesem Sinne ist der $\mathrm{EuGH}$, wie andere Verfassungsgerichte auch, ,politisch“ (Alter 2001; Dyevre 2008; allgemein zur Politisierung rechtlichen Handelns: Rehder 2007; grundlegend in Bezug auf den U.S. Supreme Court: Dahl 1957). Deshalb erscheint der Schritt von den strukturellen und akteursbezogen-soziologischen Erklärungen zur Politisierungshypothese auf den ersten Blick klein. In theoretischer Hinsicht allerdings ist der Schritt gewaltig, interpretiert die Politisierungshypothese den EuGH doch in völlig anderem Licht. Erstens schwindet der supranationale Charakter der Organisation. Die Hypothese interpretiert den EuGH als intergouvernementales Gremium nach Art des Ministerrats. Zudem, zweitens, deutet die Hypothese das Gericht nicht mehr als Leistungsorganisation des Rechtssystems. Das Gericht hört auf, dem teilsystemischen Code des Rechtssystems zu folgen, und orientiert sich am Code des politischen Teilsystems (vergleiche für eine generelle Diskussion der Übernahme teilsystemfremder Codes durch gesellschaftliche Leistungsorganisationen Schimank 2008). Das Recht wird damit nicht nur der Metapher nach, sondern tatsächlich und vollständig zur „mask of politics“ (Burley/Mattli 1993: 43).

$\mathrm{Zu}$ den integrationstheoretischen Paradigmen, dem Neofunktionalismus und dem Intergouvernementalismus, steht die Politisierungshypothese quer. Traditionell schrieben vor allem Neofunktionalisten dem Recht eine eigenständige Integrationsfunktion zu und betonten dessen Fähigkeit, politische Blockaden zu überwinden. Dabei interpretierten sie den EuGH aber als supranationales Organ im engeren Sinne, das mit privaten, an Integration interessierten Akteuren in den Mitgliedstaaten ,über Bande" spielte (Burley/Mattli 1993; Mattli/Slaughter 1998). Intergouvernementalisten wie Garrett (1995) und Moravcsik (1993) hingegen betonten, im Einklang mit der Politisierungshypothese, den zwischenstaatlichen Charakter des Integrationsprozesses.
Aber der EuGH wurde in dieser Sicht nicht selbst zur intergouvernementalen Arena politischer Aushandlungen. Vielmehr wurde er als Empfänger rationaler Delegationsakte der Mitgliedstaaten (zur Überwachung unvollständiger Verträge) interpretiert, der von seinen Prinzipalen - anders als in der Interpretation der Neofunktionalisten - effektiv kontrolliert werden konnte. Ein Ort, an dem die Mitgliedstaaten an den politischen Organen vorbei ,politics in disguise“ betreiben konnten, war im Integrationsmodell der Intergouvernementalisten weder vorgesehen noch nötig.

Die Politisierungshypothese hat mehrere Plausibilitätshürden zu nehmen, von denen keine unbesehen vorausgesetzt, aber auch keine vorschnell verworfen werden sollte. Die Hypothese setzt voraus, dass bei der Richterbestellung ,patterns of delegation“ (so Döring 2007 am Beispiel der Kommission) existieren, die darauf hinwirken, dass die Aufgaben der entsandten Richter mit der Verfolgung länder- oder parteienspezifischer Interessen ,aufgeladen“ werden. Zudem setzt die Hypothese voraus, dass die Richter die entsprechenden Interessen bei der Urteilsfindung tatsächlich auch gegen Widerstände zur Geltung bringen. Außer Frage steht, dass das institutionelle Setting, in dem sich die Richter bewegen, nicht auf effektive Kontrolle durch die Mitgliedstaaten (oder gar: durch Parteien) angelegt ist. Die Hypothese kann sich, um in den Grundzügen diskutabel zu erscheinen, nicht auf die Möglichkeit einer solchen Kontrolle stützen. Denkbar ist aber durchaus, dass die Richter die an sie herangetragenen politischen Ansprüche internalisieren und als Teil ihres intrinsischen Selbstverständnisses interpretieren.

Für die Plausibilität der Annahme eines länderbezogenen Delegationsakts spricht, dass alle EU-Mitgliedstaaten Wert darauf legen, im EuGH durch einen Richter repräsentiert zu werden. Eine etwaige Verkleinerung des EuGH auf eine die Anzahl der EU-Länder unterschreitende Richteranzahl erscheint nicht durchsetzbar und wurde im Zuge der Verhandlungen über den Verfassungsvertrag und den Lissabon-Vertrag deshalb auch nicht in Angriff genommen.

Auch die Annahme eines parteienbezogenen Delegationsakts erscheint zumindest so plausibel, dass sie nicht vorschnell verworfen werden sollte. Denn empirisch ist die Entsendung von Richtern an den EuGH tatsächlich Gegenstand parteipolitischer Auseinandersetzungen. Das lässt sich anhand des deutschen Beispiels illustrieren. Bereits vor ihrer Berufung hatte die von der rot-grünen Bundesregierung entsandte EuGH-Richterin Ninon Colneric (Amtszeit in den Jahren 2000 bis 2006) die „kraftvolle Antidiskriminierungsrechtsprechung" des EuGH gerühmt (FAZ vom 6. 6. 2006: 20) und klargestellt: „Niemand hängt seine vorherigen Einstellungen im Gericht an den Nagel" (Focus 11. 9. 2000: 37). Im Jahr 2006 setzte sich die SPD für eine zweite Amtszeit Colnerics ein. In der CDU/CSU galt Colneric aber als zu links, gewerkschaftsnah und feministisch (taz vom 7. 6. 2006: 6). Wegen ihres stärkeren Gewichts in der großen Koalition gelang es den Unionsparteien, den im Vergleich zu Colneric als eher ,unternehmerfreundlich“ (SZ vom 8. 6. 2006: 6) geltenden Thomas von Danwitz als Nachfolger durchzusetzen (Amtszeit seit 2006). Und auch die Berufung des Vorgängers Colnerics, Günter Hirsch (Amtszeit von 1994 bis 2000), war von parteipolitischem Gerangel überlagert (FAZ vom 7. 3. 2000: 16).

Eine zurückhaltende Interpretation dieser Beobachtungen würde lauten, dass parteipolitische Auseinandersetzungen über die Bestellung von Richtern ausschließlich dem Streben nach Patronage folgen - dass sie also von dem Bemühen getragen sind, parteinahen Personen zu prestigeträchtigen Ämtern zu ver-

7 Nichts anderes gilt beispielsweise für Österreich, wo die von Wolfgang Schüssel geführte konservative Regierung gegen Ende 1994 den der ÖVP nahe stehenden Peter Jann durchsetzte (Amtszeit seit 1995), der sich insbesondere mit seinem Engagement bei der Rechtsprechung gegen so genannte „Goldene Aktien“ einen Namen machte. 
helfen, ohne dass dies Wirkungen auf den materiellen Gehalt der Rechtsprechung entfalten würde. Diese Minimalinterpretation erscheint allerdings zweifelhaft, wie das Beispiel Colnerics nahe legt. Zumindest muss, so meine ich, angenommen werden, dass die Parteien bei der Auswahl von Richtern der Möglichkeit Rechnung tragen, dass die Durchsetzung ihrer Kandidaten für die Entscheidungen des Gerichts einen Unterschied machen könnte. Ob die Politisierungshypothese in einer oder beiden Varianten für den EuGH greift, ist Gegenstand der nachfolgenden Betrachtungen.

\section{Methode und Daten}

Wegen Restriktionen bei der Datenverfügbarkeit erscheint die Politisierungshypothese nicht im engeren Sinne überprüfbar. Daran ändert auch die nachfolgende Analyse nichts. Um die Hypothese zu testen, wären verlässliche Informationen zum Verhalten der europäischen Richter bei der Urteilsfindung vonnöten. Der Verlauf dieser Beratungen ist Öffentlichkeit und Forschung aber nicht zugänglich. Hinzu kommt, dass - wie bereits dargestellt - den EuGH-Urteilen keine namentlich gekennzeichneten dissenting opinions hinzugefügt werden, die über die Konfliktlinien im Richtergremium Auskunft geben könnten. Kurz: Die Richter beraten im Geheimen und vertreten die Ergebnisse der Rechtsfindung gemeinsam. Deshalb dürfen die nachfolgenden Betrachtungen auch nicht als empirischer Test im strengen Sinne verstanden werden. Immerhin aber erlaubt eine Anzahl von Hilfskonstruktionen, einige Hinweise darauf $\mathrm{zu}$ generieren, $\mathrm{ob}$ es die Politisierungshypothese wert ist, weiter verfolgt zu werden.

$\mathrm{Zu}$ diesem Zweck habe ich aus der EuGH-Rechtsprechung der 1990er und 2000er Jahre zehn Urteile als Referenzfälle ausgewählt. Die Auswahl erfolgte anhand von zwei Kriterien. Erstens handelt es sich um Fälle, die in der rechtswissenschaftlichen Literatur als rechtsfortbildend gewertet werden. Zweitens gilt für alle zehn Urteile, dass die Zuschreibung von Länderinteressen und/oder parteipolitischen Interessen an den von den Richtern behandelten Gegenständen vergleichsweise unmittelbar möglich erscheint. Weil die Anzahl an Urteilen, auf die diese zwei Kriterien zutreffen, die Anzahl an ausgewählten Fällen übersteigt, handelt es sich um eine Zufallsauswahl aus einer größeren, letztlich schwer bestimmbaren Grundgesamtheit. ${ }^{8}$ Diese Urteile werden in Abschnitt 4 eingehend beschrieben. Zudem werden dort Erwartungen an die länder- und parteipolitischen Zusammensetzungen der mit den Urteilsfindungen betrauten Kammern formuliert. Treffen diese Erwartungen $\mathrm{zu}$, ist das noch lange kein Beweis für einen tatsächlichen Politisierungsvorgang. Denn es verbleibt stets die Möglichkeit, dass der juridische Code bei der Urteilsfindung dominierte und die Zusammensetzung des Richterkreises lediglich zufällig mit den Erwartungen der Politisierungshypothese übereinstimmt. Finden sich hingegen keine entsprechenden Übereinstimmungen (oder lediglich Übereinstimmungen im Rahmen der Zufallswahrscheinlichkeit), dann spricht wenig dafür, die Politisierungshypothese im Kreis der potenziell erklärungskräftigen Deutungen der EuGH-Rechtsfortbildung zu belassen. Fünf der zehn Urteile sprechen im weiteren Sinne zum Problemfeld der wirtschaftlichen Liberalisierung (Reichweite der Grundfreiheiten), bei den anderen fünf geht es um Nichtdiskriminierung am Arbeitsplatz, Arbeitnehmerrechte und den transnationalen Zugang zu den sozialen Sicherungssystemen (einschließlich der Gesundheitssysteme) der Mitgliedstaaten. Das früheste betrachtete Urteil ist das Keck und Mithouard-Urteil vom 24.11. 1993, das späteste das Rüffert-Urteil vom 3. 4. 2008.

Der gewählten Forschungsstrategie kommt zugute, dass die personelle Zusammensetzung der Kammern von Urteil zu Urteil variiert. Nach Eingang einer Klageschrift bestimmt der Präsident des Gerichtshofs einen Berichterstatter, der je nach Rechtsgebiet und absehbarer Reichweite des Falls einen Vorschlag zur Verweisung an eine der Kammern des Gerichts oder an das Plenum formuliert. Eine weitere Ursache für Varianz in der personellen Zusammensetzung der Richterkreise besteht darin, dass den Kammern mitunter zusätzliche Richter zugeteilt werden. Der Berichterstatter nimmt selbst als Richter an der Urteilsfindung teil. Der Generalanwalt, der einen Vorschlag zur Stoßrichtung des Urteils formuliert (dem häufig, aber keineswegs immer gefolgt wird), zählt hingegen nicht zum beratenden Richterkollegium. Zwei der zehn ausgewählten Urteile wurden von einem 5er-Gremium gefällt, zwei von einem 9er-Gremium, fünf von einem $13 \mathrm{er}-\mathrm{Gremiun}$ und eines von einem 15er-Gremium (siehe Tabelle auf Seite 148).

Anhand der Urteilstexte wurden die Namen der beteiligten Richter ermittelt. Diese wurden den Ländern, aus denen die Richter entsandt wurden, zugeordnet. 25 der maximal möglichen 27 Herkunftsländer sind in dem Sample vertreten. Lediglich tschechische und bulgarische Richter waren an keinem der zehn Urteile beteiligt. Insgesamt befanden sich in den betrachteten rechtsprechenden Kammern 108 Richter (im Durchschnitt: 10,8). Diese 108 Beobachtungspunkte entfallen auf 47 unterschiedliche Personen. Österreichische, portugiesische und spanische Richter waren - mit jeweils sechs Fällen - am häufigsten an den Urteilen des Samples beteiligt. Zur Diskussion der länderbezogenen Politisierungshypothese wird in Abschnitt 5 fallweise auf weitere Hilfsvariablen zurückgegriffen.

Neben der Länderzugehörigkeit wurde der Beginn der ersten Amtszeit der Richter in die Datenbasis aufgenommen. Damit konnte recherchiert werden, welche Parteien zum Zeitpunkt der Bestellung der Richter in dem jeweiligen Mitgliedsland regierten. Wurden Richter erneut berufen (wurde also ihre Amtszeit verlängert), wurde gleichwohl die parteipolitische Zusammensetzung der Regierungen zum Zeitpunkt der Erstberufung als maßgeblich gewertet. Diese Entscheidung folgt der Einsicht, dass Wiederberufungen häufig dem Motiv der Kontinuität folgen, so dass etwaige parteipolitische Motive bei den Erstberufungen klarer zum Ausdruck kommen als bei Verlängerungen der Amtszeiten.

Auf Grundlage dieser Erhebung konnte die quasi-parteipolitische Zusammensetzung der rechtsfindenden Kammern ermittelt werden. Hierbei wurde auf den ursprünglich von Manfred G. Schmidt konstruierten fünfstufigen Index der parteipolitischen Zusammensetzung von Regierungen zurückgegriffen. Bei diesem Index steht 1 für die Hegemonie von rechts von der Mitte stehenden Parteien (sowohl konservative als auch christdemokratische Parteien), 2 für die Dominanz von Rechtsparteien, 3 für Patt-Konstellationen zwischen Links und Rechts, 4 für die Dominanz von linken oder sozialdemokratischen Parteien und 5 für deren Hegemonie. Diese Daten stammen aus zwei Versionen des Comparative Political Dataset (Armingeon, et al. 2009a und 2009b). ${ }^{9}$ Über alle 108 Beobachtungspunkte hinweg ergibt sich

8 Einen systematischen Bias der Auswahl der Fälle schließe ich aus, weil mir die Zusammensetzung der Richtergremien bei der Auswahl der Fälle noch nicht bekannt war. Nicht ausschließen kann ich allerdings einen zufällig zustande gekommenen Bias dahingehend, dass eine andere Auswahl an Fällen andere Hinweise auf die Berechtigung der Politisierungshypothese generiert hätte. Zudem bin ich Michael Blauberger für den Hinweis dankbar, dass nicht auszuschließen ist, dass Grade an Politisierung systematisch mit der Größe der Kammern variieren, weil das Gericht gerade bei brisanten Fällen besondere Vorsicht walten lassen könnte. Zur Überprüfung dieser These wäre ein Sample aus Fällen vonnöten, die ein vergleichbares Potenzial an Rechtsfortbildungen beinhalten, aber sowohl hinsichtlich der materiellen Gehalte der Urteile als auch hinsichtlich der Kammergrößen variieren.

9 Der Schmidt-Index ist der gebräuchlichste politikwissenschaftliche Index zur Messung der parteipolitischen Zusammensetzung von Regierungen entlang der Links-Rechts-Achse. Zusätzlich wurden die Kabinettsanteile der Parteien zu den Zeitpunkten der Richterentsendung in die Datenbasis eingefügt. Diese Informationen stammen aus denselben Datenquellen und haben den Vorteil, dass hier zwischen Parteien des Zentrums (nach Art der deutschen CDU) einerseits und konservativen Parteien (nach Art der britischen Tories) andererseits unterschieden wird (im Schmidt-Index werden bei- 
für die parteipolitische Zuordnung der Richter ein Durchschnittswert von 2,6, also ein leicht nach rechts verschobener Schwerpunkt.

\section{Zehn richtungsweisende EuGH-Urteile}

\subsection{Urteile zur wirtschaftlichen Liberalisierung}

Die in diesem Unterabschnitt behandelten fünf Urteile haben gemein, dass bei ihnen die (stets umstrittene) Reichweite der Grundfreiheiten im Zentrum stand. Die Grundfreiheiten zielen auf die freie Bewegung von natürlichen und juristischen Personen, Kapital, Waren und Dienstleistungen auf dem europäischen Binnenmarkt. Die Judikatur zu den Grundfreiheiten beruhte zunächst auf dem Grundsatz der Nichtdiskriminierung: Anbieter aus anderen europäischen Ländern durften auf heimischen Märkten nicht schlechter gestellt werden als Inländer. In den siebziger Jahren begann der EuGH, diese Diskriminierungsverbote in Beschränkungsverbote umzudeuten. Demnach verstoßen Beschränkungen der transnationalen Ausübung der Grundfreiheiten auch dann gegen europäisches Recht, wenn sie diskriminierungsfrei auf In- und Ausländer angewendet werden. Zwingende Gründe des Allgemeininteresses müssen vorliegen, sollen solche Beschränkungen vor dem europäischen Recht Bestand haben, und die Verhältnismäßigkeit der entsprechenden Maßnahmen muss gewahrt werden.

Diese Grundsätze entwickelte der EuGH in den siebziger Jahren mit seinen Grundsatzurteilen zu den Rechtssachen Dassonville (C-8/74) und Cassis de Dijon (C-120/78). Zunächst nur auf die Warenverkehrsfreiheit bezogen, übertrug der EuGH diese Rechtsprechung in den nachfolgenden Jahrzehnten auch auf die anderen Grundfreiheiten. Aus zwei Gründen ist die Reinterpretation der Grundfreiheiten von besonders hoher Brisanz. Erstens geht mit ihr eine deutliche Ausweitung wirtschaftlicher Freiheiten einher (wirtschaftliche Liberalisierung). Zweitens verfügt der EuGH mit den Beschränkungsverboten gewissermaßen über „Generalkompetenzen“, mit denen das Gericht auf Regelungsbestände zugreifen kann, die eigentlich in den ausschließlichen Kompetenzbereich der Mitgliedstaaten fallen. So hatte der EuGH beispielsweise im Fall Schmidberger (C-112/ 00) darüber zu befinden, ob Österreich eine von Umweltaktivisten durchgeführte Blockade der Brenner-Autobahn hätte unterbinden müssen, beschränkte diese doch zeitweilig den freien Warenverkehr. Es existiere „kein Lebensbereich“, der nicht von den als Beschränkungsverboten interpretierten Grundfreiheiten tangiert werde, formulierte in diesem Sinne der amtierende EuGH-Präsident Vassilous Skouris (Capital vom 19. 7. 2007: 46).

Eines der Politikfelder, das zwar eigentlich in den Kompetenzbereich der Mitgliedstaaten fällt, aber gleichwohl von den europäischen Grundfreiheiten erfasst und entsprechend durch EuGH-Urteile transformiert wird, ist die Steuerpolitik, speziell: die Erhebung von Ertragssteuern. Der Binnenmarkt eröffnet transnationalen Unternehmen Möglichkeiten, durch kreative länderübergreifende Verrechnungen von Gewinnen und Verlusten Strategien der Steuervermeidung zu verfolgen. In einer Serie umstrittener Urteile interpretierte der EuGH solche Strategien als legitime Nutzung der Binnenmarktregeln, beurteilte Versuche der Regierungen, solchen Strategien Grenzen zu setzen, aber als Störungen des Binnenmarktes. Im Ergebnis heizt der EuGH damit den transnationalen Steuerwettbewerb an und führt eine de-facto-Vergemeinschaftung dieses Politikfelds herbei (Ganghof/Genschel 2008: 328-329). Kritiker bemängeln den „bemerkenswerten Rigorismus“ (Heinemann/Wendt 2007: 283) der Steuerrechtsprechung des EuGH und interpretieren sie als „strukturell destruktiv“ (Drüen/Kahler 2005: 183). Eine besonders umstrittene Richtungsentscheidung, die als erstes Urteil Eingang in das hier zusammengestellte Sample findet, war das EuGH-Urteil im Fall Marks \& Spencer (C-446/03) vom 13. 12. 2005, mit dem das Gericht das britische Verbot von Verlustverrechnungen als Verstoß gegen die Binnenmarktfreiheiten verwarf. Es müsse Unternehmen gestattet werden, so der Kern des Urteils, im Ausland entstandene Verluste im Inland steuermindernd geltend zu machen. Greift die Politisierungshypothese, dann wäre zu erwarten, dass dieses Urteil mehrheitlich von Richtern aus Ländern gefällt wurde, die aufgrund ihrer niedrigen Steuersätze von Intensivierungen des europäischen Steuerwettbewerbs profitieren.

Auch das Centros-Urteil (C-212/97) vom 9. 3. 1999 ist eine besonders umstrittene Richtungsentscheidung, die im Kern wirtschaftliche Liberalisierung bewirkt. Dabei handelt es sich um das erste einer längeren Serie von Urteilen zum Gesellschaftsrecht (das Regelwerk der unternehmerischen Rechtsformen). Mit dem Centros-Urteil verwarf der EuGH die Anwendung der so genannten Sitztheorie, die besagt, dass sich die Rechtsverhältnisse einer Gesellschaft nicht nach dem Ort ihrer Gründung, sondern nach dem Urteil ihres tatsächlichen Firmensitzes richten. Befand sich also ein Unternehmenssitz in Deutschland, erlangte es nur formalen Unternehmensstatus, wenn es im Einklang mit dem deutschen Gesellschaftsrecht registriert wurde. Mit Centros und den nachfolgenden Urteilen stellte der EuGH die ausländische Briefkastenholding unter europarechtlichen Schutz. Damit werden die Mitgliedstaaten faktisch gezwungen, die Gründung von Gesellschaften mit allen in den EU-27 vertretenen Rechtsformen innerhalb ihrer Territorien zu akzeptieren, und zwar selbst dann, wenn diese nirgendwo sonst als im Inland wirtschaftlich tätig sind (Roth 2008). Damit schwindet die Fähigkeit der Mitgliedstaaten, beispielsweise eigene Regelungen zur Mindestkapitalisierung oder Mitbestimmungsregeln durchzusetzen. Insbesondere aufgrund der mittelbaren Wirkungen auf die unterschiedlichen europäischen Mitbestimmungskulturen waren die Urteile zu Centros, Überseering (C-208/00) und Inspire Art (C-167/01) umstritten. Greift die Politisierungshypothese, dann wäre zu erwarten, dass das Centros-Urteil von Richtern gesprochen wurde, die in ihrer Mehrzahl aus Ländern ohne anspruchsvolle Beteiligungsrechte der Beschäftigten auf Ebene der Leitungsorgane großer Unternehmen stammten.

Das dritte Urteil des Samples, die Laval-Entscheidung (C-341/05) vom 18. 12. 2007, ist neben der Mangold-Entscheidung (siehe unten) der wohl meist kritisierte Akt der EuGHRechtsfortbildung der vergangenen Jahre. Dieses Urteil behandelte die transnationale Arbeitnehmerentsendung und das Streikrecht. Im Kern ging es um die Frage, welche arbeitsrechtlichen Regeln die Mitgliedsstaaten Arbeitnehmern auferlegen dürfen, die in Wahrnehmung der Dienstleistungsfreiheit zeitweilig aus anderen EU-Ländern entsandt werden. Mit dem LavalUrteil überraschte der EuGH in zweierlei Hinsicht. Erstens: Er interpretierte die Entsenderichtlinie aus dem Jahr 1996 als abschließende Festlegung des Höchstmaßes an Regelungen, die den Arbeitgebern entsandter Beschäftigter auferlegt werden dürfen. Zweitens: Das Gericht überraschte mit einer Ausweitung des Grundsatzes der unmittelbaren Drittwirkung der Grundfreiheiten auf Private, in diesem Fall: Gewerkschaften. Das Gericht legte Gewerkschaften die Pflicht auf, Ausübungen der Grundfreiheiten nicht durch Arbeitskämpfe zu stören, es sei denn, diese dienten der Verfolgung zwingender Gründe des Allgemeininteresses (ausführlich: Höpner 2008; Joerges / Rödl 2008). Der Fall Laval steht gleichzeitig stellenvertretend für ein ähnlich gelagertes Urteil, das vom EuGH in identisch besetzter Kammer eine Woche zuvor gesprochen worden war. Bei Viking (C-438/05) ging es um eine unerlaubte Behinderung der Niederlassungsfreiheit durch transnationale Gewerkschaftskoordination. Beiden Fällen lag letztlich derselbe Grundkonflikt zugrunde, nämlich die von Klägern angezweifelte Legitimität des Kampfes gegen gezielte Ausnutzungen unterschiedlicher europäischer Lohnkosten und Arbeitsstandards. Greift die Politisierungshypothese, dann sollten diese Urteile von Richtern aus

de Parteien gleichermaßen als Rechtsparteien gewertet). Weil diese Informationen für die Interpretation der Befunde aber letztlich keinen Unterschied machten, wird auf ihre Berücksichtigung in der vorliegenden Darstellung verzichtet. 
Ländern gefällt worden sein, die hinsichtlich ihrer Arbeitsstandards und Lohnkosten unter dem europäischen Durchschnitt rangierten.

Das vierte Urteil, die Rüffert-Entscheidung vom 3.4.2008 (C-346/06), ist mit der Laval-Entscheidung eng verwandt, denn auch dieser Fall behandelte Probleme der Arbeitnehmerentsendung. Bei Rüffert ging es um die Frage, ob Gebietskörperschaften bei der Ausschreibung von öffentlichen Aufträgen vorsehen dürfen, dass nur Unternehmen zum Zuge kommen, die ihren Beschäftigen ein ortsübliches Entgelt zahlen. Diese Praxis verwarf der EuGH als Verstoß gegen die europäische Dienstleistungsfreiheit. Die Erwartungen in Bezug auf die Politisierungshypothese sind identisch mit jenen, die für die Fälle Laval und Viking formuliert wurden

Die fünfte in das Sample aufgenommene Richtungsentscheidung ist grundsätzlich anders gelagert. Auch sie ist als rechtsfortbildend zu interpretieren, weil der EuGH mit ihr eine vorher verfolgte Linie der Rechtsprechung verließ. Die Entscheidung ist im Vergleich zu den anderen in diesem Unterabschnitt vorgestellten vier Urteilen aber ,umgekehrt gepolt“". Mit seinen Urteilen zu den Rechtssachen Dassonville und Cassis de Dijon hatte der EuGH die Warenverkehrsfreiheit extensiv interpretiert. Jede Beschränkung des Warenverkehrs, die vom Ergebnis her gleiche Wirkungen wie eine Einfuhrbeschränkung entfaltete, konnte demnach als europarechtswidrig beanstandet werden. Sollten entsprechende Regeln gleichwohl aufrecht erhalten werden, waren die Mitgliedstaaten in der Pflicht, vor Gericht nachzuweisen, dass die Beschränkungen von zwingenden Gründen des Allgemeininteresses getragen und verhältnismäßig waren. Die Keck und Mithouard-Entscheidung vom 24. 11. 1993 (C-267/91 und C-268/91) zog dieser extensiven Interpretation der Grundfreiheiten eine Grenze ein, indem sie einen spezifischen Bereich von deren Geltung ausnahm: Verkaufsmodalitäten. So ist es den Mitgliedstaaten erlaubt, Verbote des Verkaufs unter Einstandspreisen oder Regelungen zu Ladenöffnungszeiten aufrecht zu erhalten. Über den konkreten Sachverhalt hinaus konnte Keck und Mithouard als Signal dafür gewertet werden, dass der EuGH nicht intendierte, über den Status Quo hinausgehende Überinterpretationen der Grundfreiheiten zuzulassen. Aus der Politisierungshypothese wäre die Erwartung abzuleiten, dass das Urteil von Richtern aus Ländern gesprochen wurde, die als ,koordinierte“, „organisierte“ Marktwirtschaften traditionell über einen ausgeprägten Rechtsbestand an marktkorrigierenden Regeln verfügen. Denn solche Regeln können im Unterschied zu marktbasierten Regulierungen bei expansiver Auslegung der Grundfreiheiten in Konflikt mit dem europäischen Recht geraten.

In den vorangegangenen Abschnitten wurden ausschließlich länderbezogene Erwartungen zur Zusammensetzung der rechtsfindenden Kammern formuliert. Sie sind durch Erwartungen hinsichtlich der Parteinähe der Richter zu ergänzen. Folgt man der Annahme, dass sich der Konflikt zwischen Staat (und anderen Formen der kollektiven Regulierung) und Markt entlang der politischen Links/Rechts-Achse sortiert, dann wäre zu erwarten, dass die oben dargestellten Richtungsentscheidungen von Kammern gesprochen wurden, die überdurchschnittlich mit Richtern besetzt waren, die von Mitte-Rechts-Regierungen entsandt wurden. Das gilt für alle Entscheidungen außer dem Keck und Mithouard-Urteil, bei dem ein Übergewicht ,linker" Richter zu erwarten wäre.

\subsection{Urteile zur sozialen Sicherheit von Wanderarbeitnehmern, zum Diskriminierungsschutz und zu Arbeitnehmerrechten}

In einer langen Serie von Urteilen hat der EuGH Entscheidungen zum sozialen Status von Wanderarbeitnehmern gefällt. Bei dieser Rechtsprechung leitete das Gericht aus den Grundsätzen der Arbeitnehmerfreizügigkeit, der Dienstleistungsfreiheit, der Unionsbürgerschaft und dem Diskriminierungsverbot aus Gründen der Staatsangehörigkeit Rechte zum transnationalen Zugang zu den sozialen Sicherungssystemen der Mitgliedstaaten ab. Auch diese Linie der Rechtsprechung ermöglicht die Identifikation von besonders weitreichenden (und deshalb: besonders umstrittenen) Richtungsentscheidungen. Zwei Beispiele hierfür sind das Kohll-Urteil vom 28.4. 1998 (C-158/96) und das Grzelczyk-Urteil vom 20. 9. 2001 (C-184/99). Beim Urteil in der Rechtssache Kohll ging es um Regelungen, die vorsahen, dass die Inanspruchnahme von Gesundheitsdienstleistungen im Ausland der vorherigen Genehmigung der für die Kostenerstattung zuständigen Krankenkassen bedurfte. Derartige Regelungen, so der Kern des Urteils, seien als Behinderungen der Dienstleitungsfreiheit nicht mit den Binnenmarktregeln zu vereinbaren. Ging es hier um die Pflicht inländischer Versicherungsträger, von Inländern im Ausland verursachte Kosten zu übernehmen, betraf das Grzelczyk-Urteil die Verpflichtung, von Ausländern inländisch verursachte Kosten zu tragen. Im konkreten Fall ging es um einen ausländischen Studenten, der vom belgischen Staat verlangte, das dort üblicherweise gewährte Existenzminimum zu erhalten.

Beide Urteile behandeln auf unterschiedliche Weise die Übertragung national basierter Solidarität auf transnationale Sachverhalte. Die zugrunde liegende Konfliktlinie ist widersprüchlich. Einerseits wurden diese Urteile stets als Akte der Entstehung einer dringend benötigten europäisch-transnationalen Solidarität interpretiert und entsprechend als ,soziale Rechtsprechung“ des EuGH gewürdigt (so z. B. Mayer 2009: 14-15). Das Soziale, so die optimistische Interpretation dieser Rechtsprechung, dürfe angesichts der Entstehung eines europäischen Wirtschaftsraums nicht auf nationaler Ebene verharren. Der transnationale Zugang zu den Sozialsystemen der Mitgliedstaaten sei damit die Keimzelle einer im Entstehen begriffenen europäischen Sozialpolitik (Sieveking 1997; Caporaso / Tarrow 2009). Im Sinne der Politisierungshypothese ließe sich hieraus die Annahme ableiten, dass diese Urteile von Richtern gesprochen wurden, die eher linken als rechten Parteien nahe stehen.

Dieser Deutung steht eine alternative, ungleich pessimistischere Lesart gegenüber. Die Rechtsprechung zum transnationalen Zugang zu den sozialen Sicherungssystemen, so argumentiert Scharpf (2008: 92-94), entkoppelt die Kreise der Beitragszahler und der Nutznießer. Wer die entsprechenden Leistungen nutzt, muss nicht mehr unbedingt zu deren Finanzierung beigetragen haben. Für die finanzielle Stabilität der sozialen Sicherungssysteme ist das unproblematisch, solange deren transnationale Inanspruchnahme überschaubar bleibt. Aber das Binnenmarktprogramm und die Arbeitnehmerfreizügigkeit im speziellen zielen ja gerade auf die Förderung der transnationalen Mobilität. Mittelfristig, so Scharpf, ist deshalb mit Deregulierungsdruck auf überdurchschnittlich generöse Wohlfahrtsstaaten zu rechnen. Die Profiteure dieser Rechtsprechung sind mithin jene Länder, die selbst über gezügelte Wohlfahrtsstaaten verfügen, deren Bürger aber als Wanderarbeiter oder beispielsweise als Anbieter von Gesundheitsdienstleistungen von der sozialen Infrastruktur der Nachbarländer profitieren. Diese Überlegung erlaubt die Formulierung einer länderbezogenen Erwartung: Greift die Politisierungshypothese, dann wäre zu erwarten, dass diese Urteile von Richtern gesprochen wurden, die aus Ländern mit eher unterdurchschnittlichen Sozialquoten stammen.

Bei den verbleibenden drei Urteilen erscheint lediglich die Formulierung von parteienbezogenen Erwartungen sinnvoll, nicht aber die Formulierung von länderbezogenen Erwartungen. Denn in allen drei Fällen verlaufen die Konfliktlinien quer durch die Länder und nur bedingt zwischen ihnen. Zwei der Fälle behandeln Probleme der Diskriminierung auf Arbeitsmärkten (siehe hierzu auch Höpner/Schäfer 2010). Das Mangold-Urteil vom 22. 11. 2005 (C-144/04) gilt als eines der umstrittensten EuGH-Urteile überhaupt. In diesem Urteil schöpfte der EuGH ein europäisches Verbot der Altersdiskriminierung aus - wie es im Urteil heißt - verschiedenen völkerrechtlichen Verträgen und den gemeinsamen Verfassungstraditionen der Mitgliedstaaten. Unter Verweis auf diesen allgemeinen Rechtsgrundsatz erklärte das Gericht einen Bestandteil der deutschen Hartz-Gesetze, der 
erleichterte Befristungen für ältere Arbeitnehmer vorsah, zum Verstoß gegen europäisches Recht (stellenvertretend für eine hohe Anzahl kritischer Stellungnahmen: Thüsing 2005). Das KreilUrteil vom 11. 1. 2000 (C-285 / 98) gilt als Meilenstein der europäischen Rechtsprechung zur Geschlechterdiskriminierung, weil es einen besonders sensiblen Bereich betraf. Der EuGH beanstandete, dass das deutsche Recht Frauen den Dienst bei der Bundeswehr verwehrte und ihnen lediglich den Zugang zum Sanitätsdienst (sowie dem Bereich der Militärmusik) ermöglichte. Die Kreil-Entscheidung zog eine entsprechende Änderung des Grundgesetzes nach sich.

Als zehntes Urteil wurde eine richtungsweisende Entscheidung zu Arbeitnehmerrechten in das Sample aufgenommen. Das Schmidt-Urteil vom 14. 4. 1994 (C-392/92) stärkte die Rechte der Arbeitnehmer gegenüber (neuen) Arbeitgebern im Zuge von Betriebsübergängen. Dabei handelt es sich um Inhaberwechsel von Betrieben, die häufig mit Kündigungen und Neueinstellungen einhergehen. Im konkreten Fall kündigte eine Sparkasse einer Raumpflegerin, weil sie die Reinigungsdienste einer externen Firma übertragen wollte; die Beschäftigte aber war durch den Vorgang, so entschied der EuGH, zur Arbeitnehmerin der Reinigungsfirma geworden. Die überaus heftige Kritik an diesem Urteil besagte, der EuGH habe den Begriff des Betriebsübergangs überdehnt und damit illegitime Rechtsschöpfung betrieben (im Einzelnen Egger 2008: 64-70). In den Fällen Mangold, Kreil und Schmidt wird aus der Politisierungshypothese die Erwartung abgeleitet, dass die Urteile von Richtern gesprochen wurden, die eher linken Parteien nahe stehen.

\section{Ergebnisse}

Für alle zehn Urteile wurden aus der Politisierungshypothese parteienbezogene Erwartungen abgeleitet. Aus Spalte 3 der folgenden Tabelle gehen die parteienbezogenen Zusammensetzungen der Richterkreise hervor. Das Gesamtergebnis fällt eindeutig aus: Die quasi-parteipolitische Zusammensetzung der mit der Rechtsfindung betrauten Kammern kann nicht die systematische Ursache für die rechtsfortbildende Tendenz der EuGHRechtsprechung sein. In sechs der zehn Fälle weicht der kammerbezogene Mittelwert nicht spürbar vom Gesamtmittelwert von 2,6 ab, und auch eine genauere Betrachtung der Zusammensetzung dieser sechs Kammern vermittelt kein hiervon abweichendes Bild. Das gilt für die Entscheidungen in den Rechtssachen Laval, Rüffert, Marks \& Spencer, Centros, Kreil sowie Keck und Mithouard.

In zwei Fällen liegen moderate Abweichungen vom Mittelwert vor. Der Fall Grzelczyk (transnationaler Zugang zur Sozialhilfe) wurde von einer Kammer entschieden, die mit 2,8 etwas „linker" besetzt war als der Durchschnitt. Die Richtung der Abweichung entspricht zwar der Erwartung der Politisierungshypothese, allerdings erscheint der Grad der Abweichung zu geringfügig, um aus ihr substanzielle Schlüsse zu ziehen. Auch im Fall Kohll (Geschlechterdiskriminierung) zeigt sich eine moderate Abweichung vom Mittelwert $(2,4)$ - allerdings entgegengesetzt der Erwartung der Politisierungshypothese.

In der Tat bemerkenswert ist, dass das besonders umstrittene Mangold-Urteil (Altersdiskriminierung) von der mit Abstand am weitesten links besetzten Kammer im Sample gefällt wurde. Der entsprechende Mittelwert von 3,2 liegt deutlich über dem Gesamtdurchschnitt von 2,6. Das passt recht gut zur Politisierungshypothese. Allerdings verbleibt im Sample ein ebenso bemerkenswertes Gegenbeispiel. Das Schmidt-Urteil (Geschlechterdiskriminierung) wurde von dem mit Abstand am weitesten rechts stehenden Richterkreis gefällt. Und dieser Befund ist sogar noch eindeutiger als der Befund im Mangold-Fall, denn bei Schmidt hatten von konservativen oder christdemokratischen Regierungen entsandte Richter im Gremium eine eigene Mehrheit. Kurz: Abgesehen von einem bemerkenswerten Treffer offenbart die Analyse nichts, das die parteienbezogene Variante der Politisierungshypothese systematisch stützen würde.
Zur Überprüfung der länderbezogenen Variante der Hypothese wurde in zwei Schritten vorgegangen. In einem ersten Schritt wurde erhoben, ob die Besetzung der jeweiligen Kammern im Vergleich zur Gesamtheit der Mitgliedstaaten in die erwartete Richtung verzerrt war (Spalte 2 der Tabelle informiert über die zugrunde liegenden Daten). In einem zweiten Schritt wurde betrachtet, ob die Länder, die gemäß der Hypothese ein besonders großes Interesse am materiellen Gehalt der Entscheidung hatten, im Richterkreis in der Mehrzahl waren. Eine Verzerrung im Sinne des ersten Schrittes kann die Wahrscheinlichkeit entsprechender Mehrheiten begünstigen. Der Zusammenhang zwischen beiden Beobachtungen ist aber keineswegs zwingend. So ist sogar denkbar, dass die ,interessierten Länder“ in einer Kammer im Vergleich zur Grundgesamtheit der Mitgliedsländer unterrepräsentiert waren, aber dennoch über eine Mehrheit verfügten. Das Hauptaugenmerk bei der Interpretation der Befunde liegt deshalb auf dem zweiten Schritt, der Betrachtung der Mehrheitskonstellation im Kreis der Richter.

Zunächst zur Frage der verzerrenden Besetzung der Kammern. Für sieben der zehn Urteile wurden länderbezogene Hypothesen formuliert. Bei zwei der Urteile stellt sich die Frage möglicher Verzerrungseffekte nicht, denn sie wurden im Plenum entschieden. Das gilt für die Fälle Centros (Gesellschaftsrecht) und Keck und Mithouard (Ausnahmen vom Beschränkungsverbot der Grundfreiheiten). Bei den übrigen fünf Fällen ergibt sich ein gemischtes Bild. Drei Verzerrungen erfolgten in erwarteter Richtung, zwei gegen sie. Bei der Entscheidung Marks \& Spencer (Kapitalbesteuerung) waren Länder mit niedrigen Unternehmenssteuersätzen im Vergleich zur Gesamtheit aller Mitgliedsländer minimal überrepräsentiert (Pearsons $\mathrm{r}=-0,15) .{ }^{10,}{ }^{11} \mathrm{Bei}$ der Rüffert-Entscheidung gab es eine leichte Verzerrung dahingehend, dass Länder mit niedrigen Arbeitskosten überrepräsentiert waren $(\mathrm{r}=-0,20) .{ }^{12}$ Eine recht deutliche Verzerrung im Sinne der Erwartungen zeigt sich im Fall Grzelczyk (transnationaler Zugang zu den sozialen Sicherungssystemen): Länder mit im selben Jahr niedrigen Sozialquoten waren in der entscheidenden Kammer spürbar überrepräsentiert $(\mathrm{r}=-0,39) .{ }^{13}$ In den anderen beiden Fällen aber zeigen sich Zusammenhänge entgegen der erwarteten Richtung. Beim Laval-Urteil (Streikrecht und Dienstleistungsfreiheit) waren, entgegen den Erwartungen, die Hochlohnökonomien sogar leicht überrepräsentiert $(r=0,29) .{ }^{14}$ Und bei der Kohll-Entscheidung (transnationale Kostenerstattung von Gesundheitsausgaben) war die rechtsprechende Kammer durch eine leichte Verzerrung zugunsten der Länder mit hohem Anteil öffentlicher Gesundheitsausgaben am BIP gekennzeichnet $(r=0,34) .{ }^{15}$

Reichert man diese Beobachtungen um einen genaueren Blick auf die Mehrheitsverhältnisse in den beratenden Kammern an, dann zeigt sich, dass die länderbezogene Variante der Politisierungshypothese den empirischen Test etwas besser besteht als die parteienbezogene. Nicht immer hilft der genauere Blick auf die Besetzung der Kammern bei der eingehenderen Interpretation, denn bei mehreren der zur Bewertung der Sachverhalte herangezogenen Hilfsvariablen handelt es sich um stetige Variablen, die keinen Hinweis darüber liefern, entlang welcher Schwellenwerte etwaige politische Auseinandersetzungen in den Kammern hätten verlaufen können. Aber immerhin: Nur in einem der sieben Fälle, für die länderbezogene Erwartungen for-

10 Die (schwache) negative Korrelation ist wie folgt $\mathrm{zu}$ interpretieren: Je niedriger die Unternehmenssteuern im Jahr der EuGH-Entscheidung, umso eher waren die Länder im Richtergremium repräsentiert.

11 Datenquelle: Eurostat (2009: Tabelle C.3.1.1 G). Bezugsjahr: 2005.

12 Datenquelle: Eurostat (2007: Tabelle „Arbeitskosten im Industrie- und Dienstleistungssektor"). Bezugsjahr: 2003.

13 Datenquelle: Europäische Kommission (2004: Tabelle ,Sozialschutzausgaben“). Bezugsjahr: 2001.

14 Datenquelle: Siehe vorige Fußnote.

15 Datenquelle OECD (2006: Tabelle „Total Health Expenditure as a Share of GDP“). Bezugsjahr: 1998. 
Zehn Richtungsentscheidungen des EuGH

\begin{tabular}{|c|c|c|c|c|}
\hline $\begin{array}{l}\text { Bezeichnung des Falls } \\
\text { und Datum }\end{array}$ & $\begin{array}{l}\text { Anzahl Richter, } \\
\text { beteiligte Länder }\end{array}$ & $\begin{array}{l}\text { Parteipolitische Zusammen- } \\
\text { setzungen der entsendenden } \\
\text { Regierungen }\end{array}$ & $\begin{array}{c}\text { Ergebnis zur } \\
\text { parteienbezogenen } \\
\text { Hypothese }\end{array}$ & $\begin{array}{l}\text { Ergebnis zur länder- } \\
\text { bezogenen Hypothese }\end{array}$ \\
\hline $\begin{array}{l}\text { Centros }(\mathrm{C}-212 / 97) \text {, } \\
\text { 9.3. } 1999\end{array}$ & $\begin{array}{l}15 \text { Richter: SPA, BEL, } \\
\text { NL, FRA, D, ÖT, ITA, } \\
\text { POR, DK, IRL, GB, } \\
\text { SWE, FIN, LUX, GR }\end{array}$ & $\begin{array}{l}5,3,3,1,1,3,3,1,3,1,1,5 \\
1,3,5 \text { (Mittelwert: } 2,6 \text { ) }\end{array}$ & & \\
\hline $\begin{array}{l}\text { Grzelczyk (C-184 / 99), } \\
\text { 20. 9. } 2001\end{array}$ & $\begin{array}{l}9 \text { Richter: SPA, GB, } \\
\text { DK, BEL, GR, ÖST, } \\
\text { FIN, LUX, IRL }\end{array}$ & $\begin{array}{l}5,1,3,3,5,3,1,3,1 \text { (Mittel- } \\
\text { wert: } 2,8)\end{array}$ & + & + \\
\hline $\begin{array}{l}\text { Keck und Mithouard } \\
\text { (C-267/91 und } \\
\text { C-268/91), } \\
24.11 .1993\end{array}$ & $\begin{array}{l}13 \text { Richter: DK, ITA, } \\
\text { POR, SPA, GB, GR, } \\
\text { BEL, LUX, SPA, FRA, } \\
\text { D, NL, IRL }\end{array}$ & $\begin{array}{l}4,3,1,5,1,5,1,3,5,1,1,3, \\
1 \text { (Mittelwert: } 2,6 \text { ) }\end{array}$ & & \\
\hline $\begin{array}{l}\text { Kohll (C-158/96), } \\
\text { 28. 4. } 1998\end{array}$ & $\begin{array}{l}13 \text { Richter: SPA, SWE, } \\
\text { DK, BEL, ITA, POR, } \\
\text { NL, IRL, GB, FRA, D, } \\
\text { ÖST, FIN }\end{array}$ & $\begin{array}{l}5,5,3,3,3,1,3,1,1,1,1,3, \\
1 \text { (Mittelwert: } 2,4 \text { ) }\end{array}$ & - & - \\
\hline $\begin{array}{l}\text { Kreil }(\mathrm{C}-285 / 98), \\
\text { 11. 1. } 2000\end{array}$ & $\begin{array}{l}9 \text { Richter: SPA, FRA, } \\
\text { POR, FIN, NL, DK, D, } \\
\text { SWE, BEL }\end{array}$ & $\begin{array}{l}5,1,1,1,3,3,1,5,3 \text { (Mittel- } \\
\text { wert: } 2,6 \text { ) }\end{array}$ & & keine Erwartung abgeleitet \\
\hline $\begin{array}{l}\text { Laval }(\mathrm{C}-341 / 05) \text {, } \\
18.12 .2007\end{array}$ & $\begin{array}{l}13 \text { Richter: GR, EST, } \\
\text { FIN, BEL, ÖST, DK, } \\
\text { LUX, SPA, GB, POL, } \\
\text { LIT, LET, IRL }\end{array}$ & $\begin{array}{l}5,1,3,3,3,1,3,1,5,4,3,2, \\
1 \text { (Mittelwert: } 2,7 \text { ) }\end{array}$ & & ++ \\
\hline $\begin{array}{l}\text { Mangold (C-144 / 04), } \\
\text { 22. 11. } 2005\end{array}$ & $\begin{array}{l}13 \text { Richter: ÖST, LUX, } \\
\text { NL, FIN, GB, SWE, } \\
\text { POR, SPA, BEL, UNG, } \\
\text { ZYP, MAL, SLN }\end{array}$ & $\begin{array}{l}3,3,3,3,5,5,4,1,3,4,3,1 \\
3 \text { (Mittelwert: } 3,2 \text { ) }\end{array}$ & ++ & keine Erwartung abgeleitet \\
\hline $\begin{array}{l}\text { Marks \& Spencer } \\
\text { (C-446/03), } \\
13.12 .2005\end{array}$ & $\begin{array}{l}13 \text { Richter: GR, DK, } \\
\text { ÖST, NL, FIN, ITA, } \\
\text { FRA, LUX, D, SLK, } \\
\text { EST, LET, IRL }\end{array}$ & $\begin{array}{l}5,3,3,3,3,2,1,3,5,1,1,2, \\
1 \text { (Mittelwert: } 2,5 \text { ) }\end{array}$ & & + \\
\hline $\begin{array}{l}\text { Rüffert (C-346/06), } \\
\text { 3. 4. } 2008\end{array}$ & $\begin{array}{l}5 \text { Richter: NL, POL, } \\
\text { LIT, FRA, RUM }\end{array}$ & 3, 4, 3, 1, 2 (Mittelwert: 2,6) & & ++ \\
\hline $\begin{array}{l}\text { Schmidt (C-392/92), } \\
\text { 14. 4. } 1994\end{array}$ & $\begin{array}{l}5 \text { Richter: POR, FRA, } \\
\text { BEL, SPA, D }\end{array}$ & $1,1,1,5,1$ (Mittelwert: 1,8$)$ & - & keine Erwartung abgeleitet \\
\hline
\end{tabular}

Spalte 1: Bezeichnung des Falls (Kläger im Ausgangsverfahren), Kürzel der Rechtssache und Datum des Urteils. Datenquelle: Urteilstexte.

Spalte 2: Anzahl der Richter in der Kammer bzw. im Plenum. Die Reihenfolgen der Richter (Länderkürzel) orientieren sich an den in den Urteilen genannten Reihenfolgen. Der Erstgenannte ist stets der Kammer- oder EuGH-Präsident, danach folgt der Berichterstatter, gefolgt von anderen vertretenen Kammerpräsidenten und den restlichen Richtern. Länderkürzel: BEL = Bel-

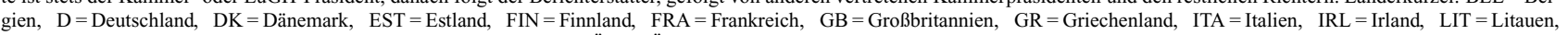
LET $=$ Lettland, LUX $=$ Luxemburg, MAL $=$ Malta, $\mathrm{NL}=$ Niederlande, ÖST $=$ Österreich, $\mathrm{POL}=$ Polen, POR $=$ Portugal, RUM $=$ Rumänien, SLK $=$ Slowakei, SLN $=$ Slowenien, SPA $=$ Spanien, $\mathrm{SWE}=$ Schweden, UNG = Ungarn, ZYP= Zypern. Datenquelle: Urteilstexte (Namen der Richter), eigene Presse- und Internetrecherchen (Länderzugehörigkeiten). ${ }^{1)}$ Besonderheit: Im Jahr 1993 erfolgte in der EG-12 die Einigung auf einen zusätzlichen, 13. Richter. Aus diesem Grund ist Spanien beim Keck und Mithouard-Urteil mit zwei Richtern vertreten.

Spalte 3: Parteipolitische Zusammensetzungen der nationalen Regierungen zu den Zeitpunkten der Amtsantritte (Erstberufungen) der jeweiligen Richter. Die Reihenfolge der Indexwerte orientiert sich an der Reihenfolge der Länder in Spalte 2. Angegeben wird der von Manfred G. Schmidt entwickelte Index der parteipolitischen Zusammensetzung von Regierungen. $1=$ Hegemonie von rechts von der Mitte stehenden Parteien (sowohl christdemokratische als auch konservative Parteien); $2=$ Dominanz von rechts von der Mitte stehenden Parteien; 3 = Patt zwischen Links und Rechts; 4 = Dominanz von links von der Mitte stehenden Parteien; 5 = Hegemonie von links von der Mitte stehenden Parteien. Datenquelle: Eigene Presse- und Internetrecherchen (Ermittlung der Zeitpunkte der Amtsantritte der Richter); Comparative Political Dataset I und III (Armingeon et al. 2009a und b) (Parteipoltische Zusammensetzungen der Regierungen).

Spalten 4 und 5: Übereinstimmungen der Ergebnisse mit den aus der Politisierungshypothese abgeleiteten Erwartungen. $++=$ Erwartungen werden deutlich bestätigt; $+=$ Erwartungen werden der Tendenz nach bestätigt; - = Ergebnisse widersprechen den Erwartungen der Tendenz nach; - Ergebnisse widersprechen den Erwartungen deutlich. Keine Angabe: Neutrale Befunde. „Keine Erwartung abgeleitet“ verweist auf die drei Fälle, in denen aus der Politisierungshypothese lediglich eine parteienbezogene, aber keine länderbezogene Erwartung folgte (vergleiche Abschnitt 4.2).

muliert wurden, läuft die vorgefundene Empirie den Voraussagen zuwider. Zwei andere Fälle sind neutral zu bewerten, zwei weitere entsprechen der Tendenz nach, und die restlichen zwei sogar deutlich den Erwartungen der länderbezogenen Politisierungshypothese.

Im Fall Kohll (transnationale Kostenerstattung von Gesundheitsausgaben) erlauben die stetigen Daten keine Bestimmung eines Schwellenwerts, entlang dessen eine politische Auseinandersetzung in der Kammer hätte verlaufen können. Die einzige verwertbare Information ist deshalb, dass die zwei Länder der damaligen EU-15, die an der Entscheidung nicht beteiligt waren, zur Gruppe der Länder mit unterdurchschnittlichen öffentlichen Gesundheitsausgaben gehörten. Diese Verzerrung widerspricht der Politisierungshypothese. Der Befund zur Centros-
Entscheidung (Gesellschaftsrecht), an der alle Länder der damaligen EU-15 beteiligt waren, ist neutral zu bewerten. Sieben der 15 Länder verfügten über Beteiligungsrechte der Beschäftigten auf Ebene der Leitungsorgane von Unternehmen, sieben andere sind als mitbestimmungsfrei $\mathrm{zu}$ klassifizieren. Ein weiteres Land, Frankreich, fungiert als „Zünglein an der Waage“. In Frankreich existieren Beteiligungsrechte, die eher symbolischer Natur sind, weil die vom Betriebsrat entsandten Vertreter im Leitungsorgan über kein Stimmrecht verfügen (Datenquelle: Höpner 2004: 40). Der Fall Centros spricht somit weder für noch gegen die Politisierungshypothese.

Mit dem Fall Grzelczyk (transnationaler Zugang zu den Sozialsystemen) verhält es sich ähnlich wie beim Kohll-Urteil. Die Bestimmung eines Schwellenwertes erscheint nicht möglich, 
immerhin war die Zusammensetzung der mit neun Richtern besetzten Kammer leicht zu Ungunsten der generösen Wohlfahrtsstaaten verzerrt. Das spricht im Zweifel für die Hypothese. Auch im Fall Marks \& Spencer (Steuerwettbewerb) ist kein Schwellenwert bestimmbar, entlang dessen sich die Mehrheitsverhältnisse in der mit 13 Richtern besetzten Kammer bestimmen ließen. Immerhin ist festzustellen, dass sieben der an der Entscheidung beteiligten Länder hinsichtlich ihrer Körperschaftssteuersätze unterhalb des Medians der damaligen EU-25 rangierten, was der Voraussage der Hypothese eher entspricht als widerspricht.

Im Fall Laval (Arbeitnehmerentsendung) zeigte sich keine im Sinne der Hypothese verzerrte Repräsentation in der mit 13 Richtern besetzten Kammer. Es lag sogar eine leichte Verzerrung in entgegengesetzter Richtung vor. Und doch offenbart der Blick auf die personelle Besetzung der Kammer eine bemerkenswerte Konstellation: Als Laval entschieden wurde, verfügten die mitteleuropäischen Hochlohnökonomien mit ihren konservativen oder sozialdemokratisch-nordischen Wohlfahrtsregimen im EuGH über keine Mehrheit mehr. Sie konnten von zwei südeuropäischen Ländern, den liberalen Ökonomien Großbritannien und Irland sowie vier osteuropäischen Beitrittsländern überstimmt werden (zur zugrunde liegenden Konfliktlinie: Schmidt 2009). Eine identische personelle Konstellation lag bei der auch inhaltlich verwandten Viking-Entscheidung vor. Noch eindeutiger ist der Befund zum Rüffert-Urteil (öffentliche Ausschreibungen). In der 5er-Kammer, die über den Fall zu befinden hatte, stellten die Vertreter Osteuropas - Polen, Litauen und Rumänien - die Mehrheit. Diese Beobachtungen entsprechen den Erwartungen der Politisierungshypothese.

Es verbleibt das Urteil zur Rechtssache Keck und Mithouard, das anders gelagert ist als alle anderen in das Sample aufgenommenen Fälle. Denn durch die Ausklammerung der Verkaufsmodalitäten vom Beschränkungsverbot im Bereich der Warenverkehrsfreiheit erfolgte keine Expansion des europäischen Rechts gegenüber nationalen Regelungen, sondern die Definition einer Grenze des Wirkungskreises einer europäischen Grundfreiheit. Mit diesem Urteil wurde deutlich, dass die Grundfreiheiten nicht uferlos genutzt werden können, um nationale Regulierungen zu Verstößen gegen die Binnenmarktregeln zu erklären und damit wirtschaftlicher Liberalisierung anheimzustellen. Die extensive Interpretation der Grundfreiheiten entfaltet eine asymmetrische Wirkung auf die Mitgliedstaaten, weil die Grundfreiheiten umso mehr mit nationalen Rechtsbeständen in Konflikt geraten, je mehr die Politischen Ökonomien der betroffenen Länder ,gemischte" Wirtschaftsordnungen sind, die über einen großen Bestand an marktkorrigierenden und -beschränkenden Regelungen verfügen (vergleiche Abschnitt 1.).

Das Keck und Mithouard-Urteil wurde im EuGH-Plenum des damaligen Europa der 12 Mitglieder gefällt, so dass sich die Frage einer verzerrten Repräsentation nicht stellt. In der damaligen EU-12 waren die „koordinierten“, „organisierten“ Ökonomien zweifellos gegenüber den liberalen Marktwirtschaften in der Mehrheit. Eine klare Bestätigung der aus der länderbezogenen Politisierungshypothese abgeleiteten Erwartungen liegt dennoch nicht vor. Denn es bliebe unerklärt, warum die Definition von Grenzen der marktschaffenden Integration trotz über lange Jahre günstiger Mehrheitsverhältnisse ein derart seltenes, in diesem Sinne sogar: einzigartiges Ereignis war.

\section{Ausblick}

Der rechtsfortbildende „Pro-Europa-Drall“ der Rechtsprechung des EuGH ist unbestritten. Strukturelle und soziologischakteursbezogene Theorien halten Erklärungsangebote für das starke Commitment bereit, das europäische Richter dem Gedanken der europäischen Integration entgegenbringen und das den EuGH zum „Motor der Integration“ hat werden lassen. Im vorliegenden Text habe ich eine alternative Deutung der Neigung des EuGH diskutiert, den europäischen Organen mehr Kompe- tenzen zuzusprechen, als es in den Verträgen vorgesehen wurde. Diese Erklärung schlägt vor, den EuGH nicht als supranationale Leistungsorganisation des Rechtssystems, sondern als intergouvernementale Aushandlungsarena $\mathrm{zu}$ interpretieren, in der das spezifisch Juridische vom politischen Code überlagert wird und die letztlich dem politischen System Europas zuzurechnen wäre. Im EuGH, so diese Sicht, können mangels Einstimmigkeitserfordernissen politische Entscheidungen fallen, die im europäischen Gesetzgebungsverfahren oder auf europäischen Gipfeln blockiert wären. Folgt man diesem Grundgedanken, verändert sich die Perspektive auf das zu klärende Puzzle radikal. Rechtsfortbildende Urteile, die faktisch Substitute für Integrationspolitik darstellen, entstehen dann nicht mehr, weil sich vom europäischen Gedanken geprägte supranationale Richter für eine mutige pro-europäische Rechtsprechung entscheiden, die mit nationalen Regelungen in Konflikt gerät. Richtungsweisende Urteile entstehen vielmehr, weil die rechtsfindenden Kammern mit Vertretern von Parteien und/ oder Ländern besetzt werden, die aufgrund parteipolitischer Nähe oder materieller Länderpräferenzen ein Interesse an den jeweiligen Urteilen haben.

Ich habe zwei Varianten der Politisierungshypothese unterschieden, eine parteienbezogene und eine länderbezogene. Die Analyse von zehn Richtungsentscheidungen des EuGH in den vergangenen zwanzig Jahren ergab keine Hinweise darauf, dass sich die parteienbezogene Hypothese zu einer ernst zu nehmenden These verdichten ließe. Immerhin bemerkenswert ist, dass das Mangold-Urteil, mit dem die Richter ein europäisches Verbot der Altersdiskriminierung schöpften, von der am weitesten "links" besetzten Kammer im Sample gesprochen wurde. Aber dieser sowie ein schwächerer Befund zum Grzelczyk-Urteil werden durch Gegenbeispiele aufgewogen, insbesondere durch das Schmidt-Urteil zur Geschlechterdiskriminierung, das von der am weitesten ,rechts“ stehenden Kammer des zusammengestellten Samples gefällt wurde. Mein Vorschlag ist deshalb, die parteienbezogene Variante der Politisierungshypothese nicht weiter zu verfolgen. So lange sich keine anders lautenden Hinweise ergeben, muss die Interpretation lauten: Das regelmäßig zu beobachtende parteipolitische Gerangel um die Entsendung von Richtern an den EuGH dient zuvörderst dem Ziel, parteinahe Juristen mit prestigeträchtigen Ämtern zu versorgen. Dass dies für die Rechtsprechung einen systematischen Unterschied machen würde, ist nicht erkennbar.

Ähnliches ließe sich von der länderbezogenen Variante der Politisierungshypothese sagen. Aus der vorliegenden Analyse folgt keine Bestätigung der Vermutung, eine etwaige Transformation des supranationalen Gerichts in eine intergouvernementale politische Aushandlungsarena ohne Einstimmigkeitszwänge sei die systematische Ursache für den Hang des EuGH zur Rechtsfortbildung. Für eine solche Interpretation war der Anteil der Fälle, bei denen sich die Erwartungen der Hypothese mehr oder weniger erfüllten, zu gering. Meine Interpretation lautet deshalb: Als systematische Erklärung der Rechtsfortbildung durch den EuGH erscheint die Politisierungshypothese nicht gut geeignet, weder in der parteienbezogenen, noch in der länderbezogenen Variante.

In einer abweichenden, abgeschwächten Variante allerdings erscheint die länderbezogene Politisierungshypothese bedenkenswert und erfährt durch die vorliegenden Ergebnisse eine gewisse Unterstützung. Die gesamte Erklärungslast der EuGHRechtsfortbildung vermag die Hypothese nicht zu tragen. Denkbar ist aber, dass in einem EuGH, dessen Richterschaft aufgrund von Eigenarten des europäischen Rechtsdiskurses eine grundsätzliche Bereitschaft $\mathrm{zu}$ einer pro-europäischen Rechtsprechung aufweist und dessen strukturelle Eigenschaften entsprechende Handlungsspielräume auch tatsächlich eröffnen, Politisierungskonstellationen von Fall zu Fall auftreten. Für diese Interpretation spricht, dass die Erwartungen der länderbezogenen Politisierungshypothese auf die Fälle Laval, Viking und Rüffert sehr gut passen. Mit größeren Abstrichen gilt das auch für die Konstellationen in den Fällen Grzelczyk sowie Marks \& Spencer. Anders als bei der parteienbezogenen Variante steht den ,Tref- 
fern" bei der länderbezogenen Variante der Hypothese keine ebenso große Anzahl an Gegenbeispielen gegenüber. In diesem Sinne verdichtet sich die länderbezogene Politisierungshypothese durch die vorliegende Betrachtung tatsächlich zu einer These, die es verdient, weiter verfolgt zu werden. Die These lautet: Akte der Politisierung des EuGH sind möglich. Der politische Code kann in die Entscheidungsfindung des Gerichts einwandern, ohne dass der rechtliche Code damit dauerhaft und vollständig vom politischen Code verdrängt würde.

In einer optimistischen Interpretation könnte dieser Befund positiv bewertet werden: Durch Einstimmigkeitserfordernisse blockierte Integrationsprojekte können sich einen Weg durch Ersatzarenen bahnen, ohne dass sie vom politischen Prozess gänzlich entkoppelt würden. Damit wird verhindert, dass integrationspolitisch unbefriedigende Lösungen wegen der spezifischen Probleme europäischer Mehrebenenpolitik dauerhaft einrasten. Vor dieser Fehlinterpretation ist zu warnen. Über den Einzelfall hinausgehende Politisierungsakte des EuGH würden die Integrationspolitik den hierfür legitimierten Entscheidungsträgern entziehen und deshalb ein das derzeitige Demokratiedefizit noch verschärfendes Legitimationsproblem erzeugen. Auch die Auflösung von Einstimmigkeitserfordernissen wäre keineswegs unbesehen positiv zu bewerten, üben sie für die EU-Mitglieder doch die wichtige Funktion des Souveränitätsschutzes aus. Sie stellen sicher, dass demokratische Selbstbestimmungsrechte nur unter Zustimmung aller Beteiligten zugunsten der europäischen Ebene aufgegeben werden können. Systematische Umgehungen von Einstimmigkeitsregeln haben deshalb ein desintegratives Potenzial. Angesichts dieser Möglichkeit bedarf die Urteilspraxis des Gerichts mehr als in der Vergangenheit der Begleitung durch eine wachsame kritische Öffentlichkeit.

Und auch über die Outcomes von Politisierungskonstellationen sollte man sich keine Illusionen machen, denn nichts spricht dafür, dass die Auflösung von Blockaden der politischen Integration den europäischen Wohlfahrtsstaat herbeiführen würde. Selbst wer eine solche Entwicklung strukturell für zumindest möglich hält, wird in Rechnung stellen müssen, dass die mitteleuropäischen Wohlfahrtsstaaten mit ihren traditionell ,gemischten" Wirtschaftsordnungen in der EU der 27, bald vielleicht 29 Mitgliedsländer über keine numerische Mehrheit ${ }^{16}$ mehr verfügen, wie die Fälle Viking, Laval und Rüffert eindrücklich zeigten. Würde der EuGH tatsächlich und über Einzelfälle hinausgehend zum Ort faktisch politischer Mehrheitsentscheidungen, würde das die Wucht der marktschaffenden Integration zusätzlich verstärken (grundlegend: Scharpf 1996), ohne dass damit neue Handlungspotenziale einer marktkorrigierenden Politik etwa im Sinne europäischer Mitbestimmungsregeln, eines europäischen Tarifvertragssystems oder europäischer Sozialversicherungen - entstehen würden.

\section{Literatur}

Alter, Karen J. (2001): Establishing the Supremacy of European Law. Oxford/New York: Oxford University Press.

Armingeon, Klaus et al. (2009a): Comparative Political Dataset I (23 OECD Countries). University of Bern.

- (2009b): Comparative Political Dataset III (35 OECD Countries and / or EU-member Countries). University of Bern.

Burley, Anne-Marie/Mattli, Walter (1993): Europe Before the Court: A Political Theory of Legal Integration, in: International Organization 47, 41-76.

Callaghan, Helen / Höpner, Martin (2005): European Integration and the Clash of Capitalisms. Political Cleavages over Takeover Liberalization, in: Comparative European Politics 3, $307-332$.

Caporaso, James A./Tarrow, Sidney (2009): Polanyi in Brussels: Supranational Institutions and the Transnational Embedding of Markets, in: International Organization 63, 593-620.
Dahl, Robert A. (1957): Decision-Making in a Democracy: The Supreme Court as a National Policy-Maker, in: Journal of Public Law 6, 279-295.

Dobler, Philipp (2008): Legitimation und Grenzen der Rechtsfortbildung durch den EuGH, in: Günter H. Roth / Peter Hilpold (Hrsg.), 509-560.

Döring, Holger (2007): The Composition of the College of Commissioners: Patterns of Delegation, in: European Union Politics 8, 209-230.

Drüen, Klaus-Dieter/Kahler, Björn (2005): Die nationale Steuerhoheit im Prozess der Europäisierung, Ii: Steuern und Wirtschaft 2005, 171-184.

Dyevre, Arthur (2008): Making Sense of Judicial Lawmaking: A Theory of Theories of Adjudication. EUI Working Paper MWP 2008/09. Badia Fiesolana: European University Institute.

Egger, Johann (2008): Nationale Interessen und Nichtdiskriminierung im Arbeits- und Sozialrecht, in: Günter H. Roth / Peter Hilpold (Hrsg.), 55-102.

Europäische Kommission (2004): Bulletin EU 4-2004. Brüssel: Europäische Kommission.

Eurostat (2007): Europa in Zahlen. Eurostat Jahrbuch 2006 / 07. Luxemburg: Amt für amtliche Veröffentlichungen der Europäischen Gemeinschaften.

- (2009): Taxation Trends in the European Union, 2009. Luxemburg: Amt für amtliche Veröffentlichungen der Europäischen Gemeinschaften.

Ganghof, Steffen / Genschel, Philipp (2008): Deregulierte Steuerpolitik: Körperschaftssteuerwettbewerb und Einkommensbesteuerung in Europa, in: Martin Höpner/Armin Schäfer (Hrsg.), Die Politische Ökonomie der europäischen Integration. Frankfurt / New York: Campus, 311-334.

Garrett, Geoffrey (1995): The Politics of Legal Integration in the European Union, in: International Organization 49, $171-181$.

van Hees, Martin/ Steunenberg, Bernhard (2000): The Choices Judges Make. Court Rulings, Personal Values, and Legal Constraints, in: Journal of Theoretical Politics 12, 305-323.

Heinemann, Friedrich/ Wendt, Carsten (2007): EU-Steuerharmonisierung auf dem Gebiet der Unternehmensbesteuerung Stand und Perspektiven, in: Integration 30, $281-291$.

Hitzel-Cassagnes, Tanja (2004): Geltung und Funktion. Supranationale Gerichtsbarkeit im Spannungsfeld von Praktischer Rationalität, Recht und Demokratie. Baden-Baden: Nomos.

Hönnige, Christoph (2007): Verfassungsgericht, Regierung und Opposition. Die vergleichende Analyse eines Spannungsdreiecks. Wiesbaden: VS Verlag.

Höpner, Martin (2004): Unternehmensmitbestimmung unter Beschuss. Die Mitbestimmungsdebatte im Licht der sozialwissenschaftlichen Forschung, in: Industrielle Beziehungen 11, $347-379$.

- (2009): Integration durch Usurpation. Thesen zur Radikalisierung der Binnenmarktintegration, in: WSI-Mitteilungen 62, $407-415$.

- (2010): Von der Lückenfüllung zur Vertragsumdeutung. Ein Vorschlag zur Unterscheidung von Stufen der Rechtsfortbil-

16 Im Gegensatz zum Rat kennt der EuGH keine für unterschiedliche Ländergrößen kompensierenden Stimmengewichtungen. Die Defensive der ,gemischten“, überdurchschnittlich großen Volkswirtschaften Kontinentaleuropas kommt deshalb im ,politisiert" gedachten EuGH stärker zum Ausdruck als im Rat oder dem europäischen Parlament. In der EU sind die Ländergröße und die Sozialschutzausgaben positiv korreliert ( $\mathrm{r}=0,39, \mathrm{n}=25$, Datenquelle: Eurostat 2007: 126, keine Angaben für Bulgarien und Rumänien), nicht etwa negativ, wie das Bild der ,small states in world markets“ (Katzenstein 1985) nahe legen würde. 
dung durch den Europäischen Gerichtshof, in: der moderne Staat 3 (im Erscheinen).

Höpner, Martin/ Schäfer, Armin (2010): Grenzen der Integration. Wie die Intensivierung der Wirtschaftsintegration zur Gefahr für die politische Integration wird, in: Integration 33, $3-20$.

Höreth, Marcus (2008): Die Selbstautorisierung des Agenten. Der Europäische Gerichtshof im Vergleich zum U.S. Supreme Court. Baden-Baden: Nomos.

Joerges, Christian / Rödl, Florian (2008): Das soziale Defizit des Europäischen Integrationsprojekts, in: Kritische Justiz 41, $149-165$.

Katzenstein, Peter J. (1985): Small States in World Markets. Industrial Policy in Europe. Ithaca/London: Cornell University Press.

Kneip, Sascha (2009): Verfassungsgerichte als demokratische Akteure. Der Beitrag des Bundesverfassungsgerichts zur Qualität der bundesdeutschen Demokratie. Baden-Baden: Nomos.

Mattli, Walter/Slaughter, Anne-Marie (1998): Revisiting the European Court of Justice, in: International Organization 52, $177-209$.

Mayer, Franz (2009): Der EuGH und das soziale Europa. Reihe Internationale Politikanalyse. Berlin: Friedrich Ebert Stiftung.

Moravcsik, Andrew (1993): Preferences and Power in the European Community: A Liberal Intergouvernmentalist Approach, in: Journal of Common Market Studies 31, 473-524.

Münch, Richard (2008): Die Konstruktion der europäischen Gesellschaft. Zur Dialektik von transnationaler Integration und nationaler Desintegration. Frankfurt/New York: Campus.

OECD (2006): Health Care Data 2006. Paris: OECD.

Pollack, Mark A. (1997): Delegation, Agency, and Agenda Setting in the European Community, in: International Organization 51, 99-134.

Pritchett, C. Herman (1948): The Roosevelt Court. A Study in Judicial Politics and Values, 1937 - 1947. New York: Macmillan.

Rehder, Britta (2007): What is Political about Jurisprudence? Courts, Politics and Political Science in Europe and the United States. MPIfG Discussion Paper 2007/05. Köln: MaxPlanck-Institut für Gesellschaftsforschung.

Robert, Anne-Cécile (2009): Die Klempner von Europa, in: Le Monde Diplomatique vom 13. 3. 2009.

Roth, Günter H. (2008): Gesellschaftsrecht: Briefkastengründungen und Golden Shares, in: Günter H. Roth / Peter Hilpold (Hrsg.), 427-479.

Roth, Günter H. / Hilpold, Peter (Hrsg.) (2008): Der EuGH und die Souveränität der Mitgliedstaaten. Eine kritische Analyse richterlicher Rechtsschöpfung auf ausgewählten Rechtsgebieten. Wien: Linde Verlag.

Scharpf, Fritz W. (1996): Negative and Positive Integration in the Political Economy of European Welfare States, in: Gary Marks et al. (Hrsg.), Governance in the European Union. London: Sage, $15-39$.

- (2008): Individualrechte gegen nationale Solidarität, in: Martin Höpner/ Armin Schäfer (Hrsg.), Die Politische Ökonomie der europäischen Integration. Frankfurt/New York: Campus, $89-99$

- (2009): Legitimität im europäischen Mehrebenensystem, in: Leviathan 37, 244-280.

Schimank, Uwe (2008): Gesellschaftliche Ökonomisierung und unternehmerisches Agieren, in: Andrea Maurer/Uwe Schimank (Hrsg.), Die Gesellschaft der Unternehmen - Die Unternehmen der Gesellschaft. Gesellschaftstheoretische Zugänge zum Wirtschaftsgeschehen. Wiesbaden: VS Verlag, 220-236.
Schmidt, Susanne (2009): When Efficiency Results in Redistribution: The Conflict Over the Single Services Market, in: West European Politics 32, 847-865.

Sieveking, Klaus (1997): Der Europäische Gerichtshof als Motor der sozialen Integration der Gemeinschaft, in: Zeitschrift für Sozialreform 43, 187-208.

Thüsing, Gregor (2005): Europarechtlicher Gleichbehandlungsgrundsatz als Bindung des Arbeitgebers, in: Zeitschrift für Wirtschaftsrecht 26, 2149-2152.

Weiler, Joseph H. (2004): A Quiet Revolution. The European Court of Justice and its Interlocutors, in: Comparative Political Studies 26, 510-534.

Wendl, Michael (2008): Kann Europa sozial werden? Kritische Anmerkungen zu einer Debatte im Magazin der Hans Böckler Stiftung. http: // www.wissentransfer.info / wissen

Wessels, Wolfgang (2008): Das politische System der Europäischen Union. Wiesbaden: VS Verlag.

\section{Umkämpfte Grenzen}

Über das Verhältnis von Sozial- und

Wirtschaftspolitik auf EU-Ebene ${ }^{1}$

\section{Wolfram Lamping}

\section{Zusammenfassung}

Begleitet von einem Diskurs der Wettbewerbsfähigkeit und Modernisierung, wird das Verhältnis von Wirtschafts- und Sozialpolitik seit den 90er Jahren auf der supranationalen Ebene neu verhandelt. Die sich in diesem Zuge herausbildende ,neue politische Ökonomie“ der EU setzt die Institutionen der Entmarktlichung, die charakteristisch für die Nachkriegssozialstaaten sind, einem erheblichen Rechtfertigungszwang und Veränderungsdruck aus. Vor diesem Hintergrund rächt es sich mittlerweile, dass die Grundfragen der sozialpolitischen Integration seit langem ihrer Beantwortung harren. Ein Aufholprozess der Sozialpolitik auf EU-Ebene scheint auch mit Blick auf den Lissabon-Vertrag unwahrscheinlich. Dieser Beitrag kommt zu dem Schluss, dass es gilt, die Frage zu klären, wie es gelingen kann, den Binnenmarkt zu vollenden, ohne die nationalen Politiken des sozialen Ausgleichs und Schutzes weiter zu beschädigen.

\section{Abstract: Contested Boundaries.}

On the Relationship between Social Policy

and Economic Policy at the EU-level

Accompanied by a debate on competitiveness and modernisation, the relationship between economic and social policy has, since the 1990s, been re-negotiated at the supranational level. The emerging 'new political economy' of the EU has constantly increased the pressure on welfare state institutions to justify and adjust their activities. Given that precarious situation, one of the enduring problems is that core questions surrounding the integration of social policies have not been sufficiently answered and addressed at the supranational level. A process of catch-up between social policy integration and economic integration at the EU level seems to be very unlikely in the coming years. In addition, the Lisbon Treaty does not provide the basis for any significant developments in this regard. This article comes to

1 Für wertvolle Hinweise und Kommentare danke ich den beiden anonymen Gutachtern. 